\title{
Presentation of menopausal symptoms: A village based community study
}

\author{
Mona Srivastava ${ }^{1}$, Ritu Srivastava ${ }^{2}$, Balram Pandit ${ }^{1}$ \\ ${ }^{1}$ Associate Professor, ${ }^{3}$ Senior Resident, Department of Psychiatry, Institute of Medical Sciences, Banaras Hindu University, Varanasi (UP), \\ India, ${ }^{2}$ Consultant Obstetrician and Gynaecologist, Maternity Health Care Centre, Gorakhpur (UP), India
}

\section{A B S T R A C T}

Background: The present observational, cross sectional study was carried out in rural women $(n=117$ ) from the Varanasi area with natural menopause to evaluate menopausal symptoms in women above the age of 40 , as well as to evaluate the correlation of age on these symptoms. Materials and Methods: A crossectional assessment by interviewing regarding the menopausal complaints in the following $40-44(n=27), 45-50(n=30)$ and above $50(n=60)$ years age groups. Menstrual rating scale (MRS) was administered to all the women forming the sample. Results: Mean age at menopause was 47.35 years. Mean number of menopausal symptoms in three age groups were as (mean \pm SD) 10.53 \pm 7.33 , $7.70 \pm 6.76$ and $14.50 \pm 10.77$ respectively, which varied significantly $(F=4.86, d f=2$, $87, P=0.009$ ). The study reveal, varying nature of symptoms with age and MDSM (Mean Duration since Menopause), with vasomotor symptoms being more prevalent with lesser MDSM and psychological and rheumatic complaints more prevalent with increasing age and MDSM in this region. Conclusion: Such regional studies will help to corroborate data so that health care providers can plan strategies for the middle aged women suffering from these menopausal symptoms.

Key Words: Menopause; Symptoms; Menstrual rating scale (MRS); Community.

\section{INTRODUCTION}

The World Health Organization (WHO) defines menopause as 'the permanent cessation of menstruation as a result of the loss of ovarian activity'. ${ }^{1}$ Menopausal period has an important role in the reproductive life of a woman and gives rise to many physical and mental problems. ${ }^{2}$ With the increasing life expectancy a women spends almost a third of her life in menopause. ${ }^{2}$ Menopause is recognized by all women in all cultures as cessation of menstruation for one year. Menopause can thus be said to be a universal reproductive phenomenon. ${ }^{3}$ Menopause has become an important subject in recent years it is generally believed that menopause is welcomed as a favorable event among rural women in India unlike in the West. ${ }^{4}$ This is attributed to the many perceived benefits of menopause such as freedom from cultural restrictions imposed on younger women and the burden of childbirth as well as the discomforts associated with menstruation. Postmenopausal women in India are said to enjoy a higher social status assigned to ageing women. ${ }^{5}$ Changes in menopause experience for women in different parts of the world and in different ethnic groups provide evidence for specific cultural and ethnic impacts of menopause. ${ }^{5}$ As is the case in many developing countries, it is difficult for women living in rural India to obtain access to healthcare services, as a result of a dearth of trained health care professionals at the grass root level and the specialized sector. The economic factors associated with the geographical diversity make commuting difficult so as to access the health care facility in the cities. ${ }^{6}$ Medical opinion has always projected menopause as a malady because of its association with a variety of acute and chronic conditions, both physical and psychological, ranging from hot flushes to more severe cardiovascular and bone diseases. ${ }^{7}$ Numerous physical and psychological symptoms have been attributed to the hormonal changes of menopause. ${ }^{7,8}$ This reproductive landmark is not always the same for all women in all cultures. ${ }^{5}$ The prevalence 
of menopausal symptoms varies widely not only among individuals of the same population but also between different ethnic populations. ${ }^{5,7}$ There is a great diversity in nature of symptom and frequencies across countries, even in the same cultures. ${ }^{5}$ Mean age at menopause ranges in Indian women from 40.32 to $48.84 \mathrm{yrs}^{9}$ and in developed countries from 48.0 to $51 \mathrm{yrs}^{2}$ Studies have been undertaken in past to find out correlation of age and symptomatology of menopause. ${ }^{9}$ Large efforts are required to educate and make these women aware of menopausal symptoms. This will help in early recognition of symptoms, reduction of discomfort and fears and enable to seek appropriate medical care if necessary. Studies on issues relating to menopause, especially among rural women, are lacking in India. We aimed to study the perceptions regarding menopause and the prevalence of menopausal symptoms among women in rural north eastern part of India.

\section{STUDY DESIGN}

The present study is an observational, cross sectional rural community based study. All women above the age of 40 years were included in the study. Women with history of using oral contraceptives pill/HRT(hormone replacement therapy), phytoestrogens, women who had attained premature or surgical menopause or those having any serious disease were excluded from the study. Study site was the community outreach centre of the community medicine department of the institute of medical sciences, Banaras Hindu University. Details of the village are as in (Table 1).

\section{MATERIALS AND METHOD}

Face to face interview of the women were conducted by all authors. Data was collected regarding socio-demographic status, medical history and climacteric symptoms. Menopausal Rating Scale (MRS $)^{10}$ was administered to all the respondents. The scale is an eleven item tool which has been widely used in clinical studies, is easy to administer and is rated on a mild, moderate, severe score. Depending upon the response, the total score is calculated and the

\begin{tabular}{lc} 
Table 1: Socio-demographic features of the rural \\
community (Tikari village) \\
\hline Population & 8132 \\
Number of females & 3847 \\
Literacy rate & $82 \%$ \\
Average size of each family & $5-6$ \\
Average income of each family & Rs $1300-2400$ \\
Main occupation of the village & Vegetable cultivation \\
Number of economically independent & $86 \%$ \\
females & \\
\hline
\end{tabular}

menopausal symptoms are estimated. Each item was read out and the women were asked whether they experienced the symptoms mentioned. The study was given the ethical clearance from the institute ethical committee. Computer software Microsoft Excel for windows and SPSS 16.0 for windows was used for analysis.

\section{RESULTS}

Data was analyzed for frequency of symptom and mean number of menopausal symptoms and these were compared in the three age groups namely, group I 40-45 yrs, group II 45-50 yrs, group III >50 yrs. Data was presented as percentages for qualitative variable. For quantitative variable mean and standard deviations were calculated. Statistical significance between the three age groups was assessed by the use of one way ANOVA and nonparametric equivalent Kruskal Wallis test. Barferrani' $t$ ' test was applied after ANOVA to assess which group varied significantly. A p value of $0.008(t, 2.62)$ was considered critical for the statistical significance. The study population comprised of 117 menopausal (Table 2) women with 23.07, 25.64 and $51.28 \%$ being enrolled in 40-44 years, $45-50$ years and $>50$ years age group respectively. Mean age at menopause

\begin{tabular}{|c|c|c|}
\hline & Number & $\%$ \\
\hline \multicolumn{3}{|l|}{ Age } \\
\hline 40-44 years & 27 & 23.07 \\
\hline $45-50$ years & 30 & 25.64 \\
\hline$>50$ years & 60 & 51.28 \\
\hline Total & 117 & \\
\hline Mean age at menopause & 47.35 years & \\
\hline \multicolumn{3}{|l|}{ Marital status } \\
\hline Married & 98 & 83.76 \\
\hline Divorced and widowed & 19 & 16.24 \\
\hline \multicolumn{3}{|l|}{ Educational status } \\
\hline Literate & 80 & 68.37 \\
\hline Illiterate & 37 & 31.63 \\
\hline \multicolumn{3}{|l|}{ Sexual activity } \\
\hline Active & 38 & 32.47 \\
\hline Inactive & 79 & 67.53 \\
\hline \multicolumn{3}{|l|}{ Life style } \\
\hline Active & 70 & 59.82 \\
\hline Hectic & 30 & 25.64 \\
\hline Sedentary & 17 & 14.54 \\
\hline \multicolumn{3}{|l|}{ Dietary habit } \\
\hline Vegetarian & 71 & 60.68 \\
\hline Mixed & 14 & 11.96 \\
\hline Non vegetarian & 32 & 27.35 \\
\hline Tea & 80 & 68.37 \\
\hline Alcohol & 1 & 1.17 \\
\hline Tobacco chewing & 14 & 11.96 \\
\hline Smoking & 10 & 11.7 \\
\hline None & 12 & 7.8 \\
\hline \multicolumn{3}{|l|}{ Effect of menopause } \\
\hline Negative & 90 & 76.92 \\
\hline Not affected & 27 & 23.08 \\
\hline
\end{tabular}


was 47.35 years. Out of total women enrolled in the study $83.76 \%$ were married and $16.24 \%$ were divorced/ widowed, whereas $68.37 \%$ of them were literate and only $31.62 \%$ were Illiterates(education being defined as the ability to read and write). Most of women (59.82\%) had an active or hectic (25.64\%) life style and only $14.52 \%$ had sedentary lifestyle. On enquiry about dietary patterns $60.68 \%$ were vegetarian, $27.35 \%$ were non-vegetarian and $11.96 \%$ were on mixed diet. $68.37 \%$ gave history of regular consumption of tea, $1.41 \%$ among total population were alcoholics. $2.56 \%$ gave history of chewing tobacco and $1.85 \%$ had the habits of smoking. On interview $76.92 \%$ of the menopausal women felt firmly that they were affected by menopause in a positive manner and only $23.03 \%$ felt that they were affected negatively by menopause. Mean number of menopausal symptoms in three age groups were as (mean \pm SD) $10.53 \pm 7.33,7.70 \pm 6.76$ and $14.50 \pm 10.77$ in 40-44 years, 45-50 years and $>50$ years age groups respectively (Table 3). During transition of menopause and in postmenopausal period the numbers of symptoms were more and in-between numbers of complaints were less. Statistically significant variation was observed in between $45-50$ years and $>50$ years age groups $(t=3.10, p=.002$, HS). Fatigue \& lack of energy $(72.93 \%)$, headache $(55.9 \%)$, hot flushes, cold sweats, cold hand and feet $53.86 \%$ each and weight gain $(43.13 \%)$ were most frequently complained menopausal symptoms in the present study.

\section{DISCUSSION}

The present study revealed mean age at menopause to be 47.35 years. Mean number of menopausal symptoms were $10.53,7.70$ and 14.50 in the 40-44, 45-50 and above 50 years age groups respectively. Most frequent menopausal symptoms were fatigue \& lack of energy $(72.93 \%)$ followed by headache $(55.9 \%)$, hot flushes, cold sweats, and cold hand and feet $(53.86 \%)$ weight gain $(43.13 \%)$. Vasomotor symptoms being more prevalent with lesser MDSM (mean duration since menopause) and psychological and rheumatic complaints more prevalent with increasing age and MDSM. The most frequent menopausal symptoms in the age group of 40-44 years with mean duration since menopause(MDSM=2.31 years) were fatigue, lack of

\begin{tabular}{lcl}
\multicolumn{3}{l}{ Table 3: Mean number of menopausal symptoms } \\
\hline $\begin{array}{l}\text { Age } \\
\text { distribution }\end{array}$ & $\begin{array}{c}\text { Mean no. of menopausal } \\
\text { symptoms (mean } \pm \text { SD) }\end{array}$ & Barferrani 't' test \\
\hline $\begin{array}{l}\text { Group I } \\
\text { (40-44 years) }\end{array}$ & $10.53 \pm 7.33$ & $\begin{array}{l}\text { Group I vs Group II } \\
\mathrm{t}=1.29, \mathrm{p}=0.19, \mathrm{NS}\end{array}$ \\
$\begin{array}{l}\text { Group II } \\
\text { (45-50 years) }\end{array}$ & $7.70 \pm 6.76$ & $\begin{array}{l}\text { Group II vs Group III } \\
\mathrm{T}=3.10, \mathrm{p}=0.002, \mathrm{HS}\end{array}$ \\
$\begin{array}{l}\text { Group III } \\
\text { (>50 years) }\end{array}$ & $14.50 \pm 10.77$ & $\begin{array}{l}\text { Group I vs Group III } \\
\mathrm{t}=1.81, \mathrm{p}=0.07, \mathrm{NS}\end{array}$ \\
ANOVA & $\mathrm{F}=4.86, \mathrm{df}=2.87, \mathrm{p}=0.009$ \\
\hline
\end{tabular}

energy (88.8\%), headache (77.7\%), hot flushes, cold sweats, cold hand and feet, numbness/tingling and excitability/ anxiety $66.6 \%$ each respectively. In the age group of $45-50$ years (MDSM=3.70 years), fatigue, lack of energy $(70 \%)$, cold hand and feet $(60 \%)$, hot flushes, cold sweats, weight gain, irritability and nervousness (50\%) were common complaints. Whereas, rheumatic pains, fatigue, lack of energy $(60 \%)$ followed by headache, pain in back, forgetfulness, neck and skull pain (50\%), sleep disturbance and depression (45\%) were frequent symptoms in the age group $>50$ years with MDSM $=8.15$ years.

A wide range in mean age at menopause has been found in Indian women from 40.32 to 48.84 yrs and in developed countries from 48.0 to 51 yrs. ${ }^{4,5,8}$ Mean age at menopause in Indian women is less in comparison to women from developed countries. These diversities may probably be because of regional, community and ethnic variations. Mean age at menopause in the present study corresponded with Singh and Arora, ${ }^{4}$ Bagga et al, ${ }^{9}$ and Kim et al. ${ }^{11}$ Numbers of symptoms were consistently more in early and late menopause period in the present study. Similar trends were observed by a study which looked at the age and symptoms of menopausal women, ${ }^{9}$ however this observation differs from other studies done in other set ups. ${ }^{12,13}$ The possible explanation could be that with transition of menopause women are most distressed, which is relatively earlier in Indian women. ${ }^{9}$ They start recognizing and coping up with these menopausal symptoms with the passage of time. ${ }^{11}$ However in postmenopausal period with complete cessation of hormone release, the menopausal complaints worsen in both severity and frequency. ${ }^{14}$ Common menopausal symptoms vary when compared with other reports from India and abroad. ${ }^{7,8}$ These diversities probably exist because women experience reduction in estrogen levels in a wide variety of ways with great inter-individual variations. ${ }^{14}$ Our study reveals that lesser the age at menopause and MDSM, more are the vasomotor symptoms, this finding is concurrent with another study. ${ }^{14}$ This is consistent with the fact that vasomotor symptoms are experienced even before the actual cessation of menstruation as a result of hypothalamic-pituitary dysfunction in response to ovarian hormonal failure. ${ }^{14}$ Thus, vasomotor symptoms are not as infrequent as previously thought to be among the rural women in India. ${ }^{4}$ A clinic-based study from Mumbai ${ }^{6}$ reported that $25 \%$ of urban women between 40 and 60 years of age complained of vasomotor symptoms. Declining ovarian functions have also been reported to cause frequency of micturition, urge incontinence, dysuria and recurrent urinary tract infections. ${ }^{14}$ Psychological and rheumatic complaints are prevalent with increasing age and MDSM. Psychological and rheumatic complaints are major features in late menopauses as reported in American women and also in Indian women. ${ }^{9}$ Despite 
the prevalence of physical problems associated with menopause; interviews revealed that half the population found cessation of menstruation to be very convenient. ${ }^{15}$ None of them appreciated any changes in their social status, i.e. renewed freedom and influence in their families thought to be associated with menopause. ${ }^{15}$ The women who reported a diminished ability to work after menopause attributed it to ageing. There was a significant prevalence of sexual dysfunction among postmenopausal women that they attributed to ageing, culture, presence of adolescent children at home and lack of privacy in traditional rural homes. ${ }^{4,15}$ We would like to conclude by stressing that such regional studies not only create awareness but also help in education of women regarding an early identification of common menopausal symptoms. These kinds of studies can also provide an understanding regarding the feasibility and need of hormone replacement therapy (HRT) in the rural community set up. ${ }^{5}$ Our study has some limitations like a lack of hormonal estimation and an absence of a clinic based assessment so as to provide a good comparison. Future studies may address both the above features.

\section{REFERENCES}

1. World Health Organization. Research on the menopause in the 1990s: Report of a WHO Scientific Group. World Health Organ Tech Rep Ser 1996;866: 1-107.

2. Us Census Bureau 1996 Global aging into the 21 st century-The wall chart by US.

3. Lock M. Contested meanings of the menopause. Lancet 1991; 337: 1270-1272.
4. Singh A and Arora AK. Profile of menopausal women in rural north India. Climacteric 2005; 8(2):177-184.

5. Obermeyer CM. Menopause across cultures: a review of the evidence. Menopause 2000; 7(3):184-192.

6. Shah R, Kalgutkar S, Savardekar L, Chitlang S, Iddya U and Balaiah D. Menopausal symptoms in urban Indian women. Obs \& Gynae Today 2004; 11(10):667-670.

7. Schnatz PF, Banever AE, Greene JF and O'sullivan DM. Pilot study of menopause symptoms in a clinic population. Menopause 2005; 12(5):623-629.

8. Malacara JM, Canto de Cetina T, Bassol S, Gonzalez N, Cacique L, Vera-Ramirez ML, et al. Symptoms at pre and post menopause in rural and urban women from three States of Mexico. Maturitas 2002; 43(1):11-9.

9. Bagga A. Age and symptomatology of menopause: a case study. Obs \& Gynae Today 2004; 11(10):660-666.

10. Berlin Z. MRS - Menopause Rating Scale. (Online)Available: http:// www.menopause-rating-scale.info/(Accessed 12 May 2013).

11. Kim YH, Ha EH and Shin SJ. A study on the menopausal symptoms and quality of life in middle aged women. Taehan Kanho Hakhoe Chi 2003; 33(5):601-608.

12. Kakkar V, Kaur D, Chopra K, Kaur A and Kaur IP. Assessment of the variation in menopausal symptoms with age, education and working/non-working status in north-Indian sub population using menopause rating scale (MRS). Maturitas 2007; 57: 306-314.

13. Aaron R, Muliyil J and Abraham S. Medico-social dimensions of menopause: A cross-sectional study from rural south India. Natl med J Ind 2002;15(1):14-17.

14. Brown WJ, Mishra GD and Dobson A. Changes in physical symptoms during the menopause transition. Int $\mathrm{J}$ Behav Med 2002; 9(1):53-67.

15. Kaur S, Walia I and Singh A. How menopause affects the lives of women in suburban Chandigarh, India. Climacteric 2004; $7(2): 175-180$.

\footnotetext{
Authors Contribution:

MS - concept,design, literature search,formatting,data collection, analysis, manuscript prepration, gurantor; RS - design,literature search, data collection, manuscript prepration; BM - literature search, data collection, clinical study.
}

Source of Support: Nil, Conflict of Interest: None declared. 